\title{
Two-stage chaotic Colpitts oscillator
}

Tamasevicius, Arunas; Mykolaitis, G.; Bumeliene, S.; Cenys, Antanas; Anagnostopoulos, A. N.; Lindberg, Erik

\author{
Published in: \\ Electronics Letters
}

Publication date:

2001

Document Version

Publisher's PDF, also known as Version of record

Link back to DTU Orbit

Citation (APA):

Tamasevicius, A., Mykolaitis, G., Bumeliene, S., Cenys, A., Anagnostopoulos, A. N., \& Lindberg, E. (2001). Twostage chaotic Colpitts oscillator. Electronics Letters, 37(9), 549-551.

http://ieeexplore.ieee.org/iel5/2220/19919/00920906.pdf

\section{General rights}

Copyright and moral rights for the publications made accessible in the public portal are retained by the authors and/or other copyright owners and it is a condition of accessing publications that users recognise and abide by the legal requirements associated with these rights.

- Users may download and print one copy of any publication from the public portal for the purpose of private study or research.

- You may not further distribute the material or use it for any profit-making activity or commercial gain

- You may freely distribute the URL identifying the publication in the public portal

If you believe that this document breaches copyright please contact us providing details, and we will remove access to the work immediately and investigate your claim 
straints on the feasible set of the objectives. An initial population size of 50-100 and up to 200 generations were tested. Various parameter values such as crossover and mutation probabilities were also examined. Results showed that convergence of the model was steady and fast. Deterministic results were also examined and achieved.

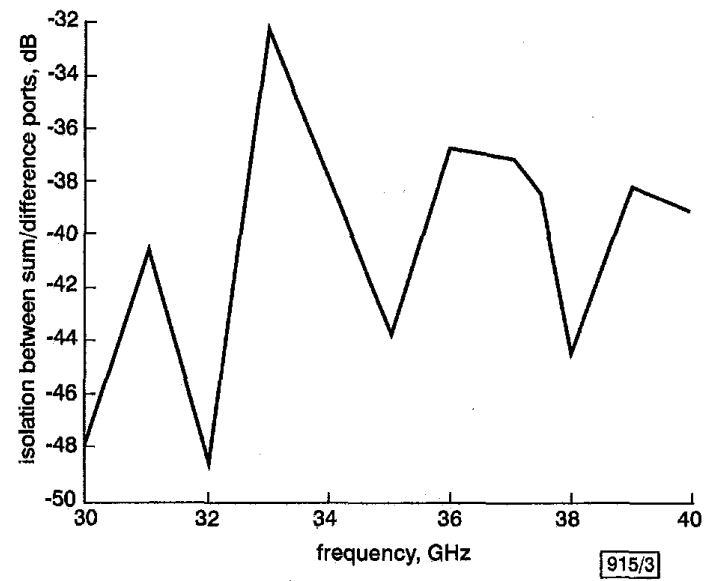

Fig. 3 Measured frequency response of isolation

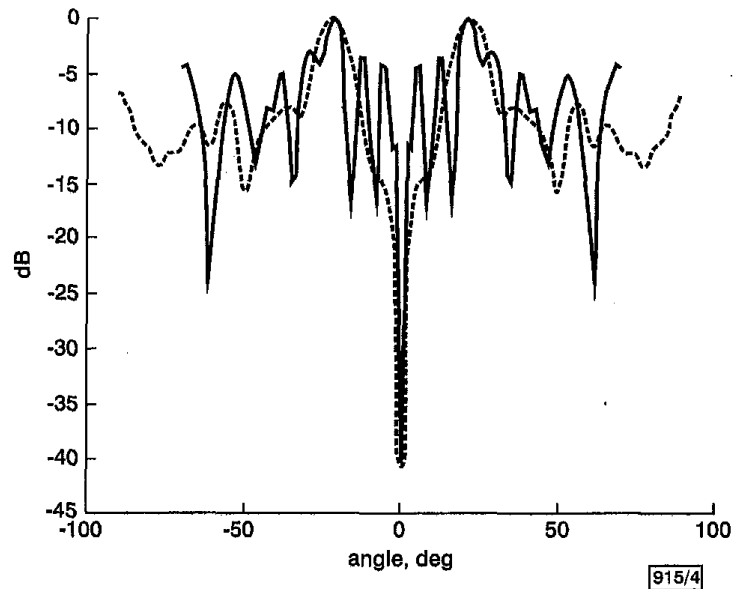

Fig. 4 Patterns of difference-beam at $37.5 \mathrm{GHz}$

- measured results

---- computed results

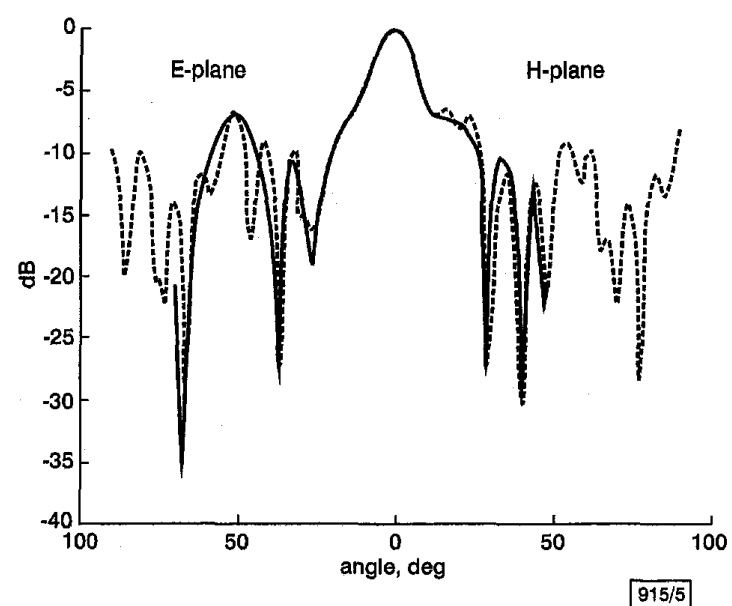

Fig, 5 Patterns of sum-beam at $37.5 \mathrm{GHz}$

-_._- measured results

-.. - computed results

Results: The optimised results were a population of chromosomes with matching objective values. However, because of the computational complexity and dependence on the feeding system, the frequency response of the input VSWR was not considered as an objective. Thus, the results were also determined according to the travelling-wave mechanism of the TSA [3]. The optimised and measured sample is shown in Fig. 1. The measured frequency responses of the VSWR at the sum/difference ports and the isolation between the two ports, which are the accumulated results of the reflection and cross-coupling of the antenna, the printed duplexer, and the microstrip-waveguide transitions, are shown in Figs. 2 and 3, respectively. The frequency bands for a VSWR $<2: 1$ were $34.5-40+\mathrm{GHz}$ at the sum-port and $33-40+\mathrm{GHz}$ at the difference-port. The band for an isolation $<36 \mathrm{~dB}$ was $33.5-40+\mathrm{GHz}$. The computed and measured radiation patterns of the differencebeam and the sum-beam at the central frequency $37.5 \mathrm{GHz}$ are shown in Figs. 4 and 5, respectively. The null-depth of the difference-beam is better than $-40 \mathrm{~dB}$ with a null-slant of $\pm 21^{\circ}$. The $-3 \mathrm{~dB}$ beamwidth of the sum-beam was $10 \times 15^{\circ}$ (for the $\mathrm{E}-\times \mathrm{H}$ - plane). In addition, the measured gain of the sum-beam was $14 \mathrm{~dB}$. Patterns were also measured at 35 and $40 \mathrm{GHz}$. The results of the difference-beams were a null-depth of $-28 \mathrm{~dB}$ with $26^{\circ}$ null-slant and $-27 \mathrm{~dB}$ with $48^{\circ}$, respectively; steep slopes at the beam-centre were also obtained. The results of the sum-beams were $-3 \mathrm{~dB}$ beamwidth of $12 \times 15^{\circ}$ and $14 \times 26^{\circ}$, and a gain of 12 and $10 \mathrm{~dB}$, respectively. The measured sidelobe levels were satisfactory. The measured cross-polarisation levels were sufficiently low and are not presented here for the sake of brevity.

Conclusions: Although the linear-edged CTSA samples in our previous research work produced good VSWR and isolation properties, they were not capable of maximising the slope of the difference beam in a wide band. Although the optimised CTSA configuration appears to be counterintuitive, it performs better in terms of the desired aspects. With the fast development of computers, more refined objectives can be taken into consideration. Therefore, further improvements in terms of both the sum/difference beams and impedance matching are still possible.

() IEE 2001

Electronics Letters Online No: 20010315

14 March 2001 DOI: $10.1049 / \mathrm{el}: 20010315$

K. Kang, W.X. Zhang and J.J. Li (State Key Laboratory of Millimeter Waves, Southeast University, Nanjing, 210096, People's Republic of China)

\section{References}

1 YIN, M., KANG, K., and ZHANG, W.X.: 'Sum/difference beam duplexer for coupled tapered slot-line antenna', Electron. Lett., 2000, 36, (11), pp, 935-936

2 BARRETT, R., BERRY, M., CHAN, T.F., DEMMEL, J., DONATO, J., DONGARRA, J., EIJKHOUT, v., POZO, R., ROMINE, C., and VAN DER VORST, H.: "Templates for the solution of linear systems; building blocks for iterative methods' (SIAM Press, Philadelphia, PA, 1994)

3 YNGVESSON, K.S., KORZENIOWSKI, T.L., KIM, Y., KOLLBERG, E.L., and JOHNANSSON, J.F.: 'The tapered slot antenna - a new integrated element for millimeter-wave applications', IEEE Trans, Microw. Theory Tech., 1989, 37, (2), pp. 365-374

\section{Two-stage chaotic Colpitts oscillator}

A. Tamaševičius, G. Mykolaitis, S. Bumelienè, A. Cenys, A.N. Anagnostopoulos and E. Lindberg

A novel version of the chaotic Colpitts oscillator is proposed. It contains two bipolar junction transistors coupled in series. The resonance loop consists of an inductor and three capacitors. The two-stage oscillator, compared with the classical circuit, enables the fundamental frequency of chaotic oscillations to be increased by a factor of three. The PSpice simulations performed with 9 $\mathrm{GHz}$ threshold frequency transistors demonstrate that the highest fundamental frequencies of chaotic behaviour are 1 and $3 \mathrm{GHz}$ for the classical and the two-stage Colpitts oscillator, respectively.

Introduction: Classical oscillators such as the Colpitts, the Hartley and the Wien-bridge, are commonly used to generate periodic waveforms. However, with special sets of circuit parameters and 
circuitry modifications these oscillators can exhibit chaotic behaviour. For example, the classical Colpitts oscillator (Fig. 1) with a bipolar junction transistor (BJT) has been demonstrated to generate chaos at the fundamental frequency $f^{*} \approx 100 \mathrm{kHz}$ [1]. Various features of the chaotic Colpitts oscillator have been considered in $[2,3]$. Chaos in the Colpitts oscillator has also been studied in the HF range. Using the general purpose $2 \mathrm{~N} 2222 \mathrm{~A}$ type BJT with threshold frequency $f_{T}=300 \mathrm{MHz}$, chaotic oscillations have been observed at $f^{*}=25 \mathrm{MHz}$ [4]. The PSpice simulations indicate that for chaotic oscillations the highest $f^{*}$ achievable with the 2N2222A type transistor is $\sim 30 \mathrm{MHz}$, i.e. ten times less than the $f_{T}$. Meanwhile periodic oscillations can be generated at frequencies very close to the $f_{T}$. In the UHF range chaos has been predicted in the Colpitts oscillator with the Avantek AT41486 type BJT $\left(f_{T}=\right.$ $3 \mathrm{GHz}$ ) by means of PSpice simulations at $f^{*}=500 \mathrm{MHz}$ [4]. Very recently, the Colpitts oscillator has been shown experimentally to generate chaos in the UHF range [5]. Employing a 2T938A-2 type transistor (Russian $n-p-n$ silicon BJT with $f_{T} \approx 5 \mathrm{GHz}$ ) and a microstrip line based distributed resonator, much more complicated than the L-C1-C2 in Fig. 1, the authors of [5] have demonstrated narrowband $(\Delta f=10 \%)$ chaos in the UHF range at $f^{*}=$ 900 to $1000 \mathrm{MHz}$

In this Letter we propose a novel version of the chaotic Colpitts oscillator, specifically a two-stage circuit. The modified circuit, compared with the classical Colpitts oscillator, is shown to move the highest fundamental frequency $f^{*}$ from approximately 0.1 to $0.3 f_{T}$, i.e. essentially closer to the threshold frequency $f_{T}$.

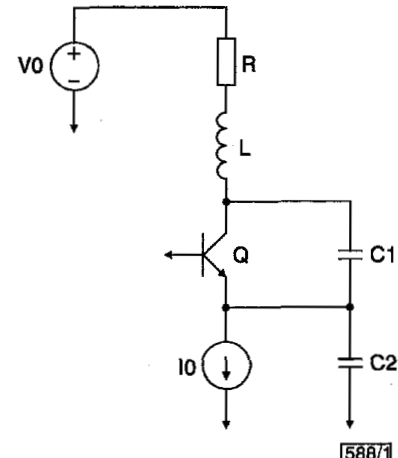

Fig. 1 Circuit diagram of classical Colpitts oscillator

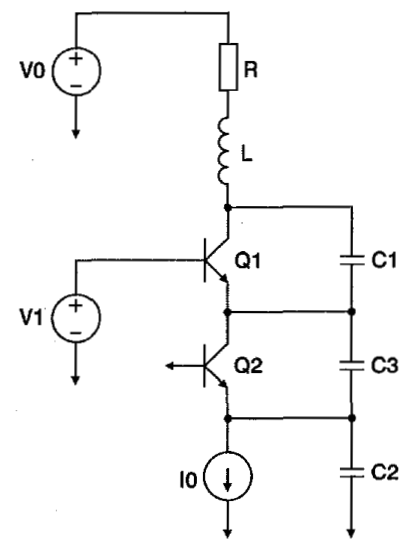

$588 / 2$

Fig. 2 Circuit diagram of novel two-stage Colpitts oscillator

Modified circuit: The circuit diagram of the novel chaotic oscillator is presented in Fig. 2. Compared with the classical oscillator (Fig. 1) it contains an extra transistor, Q2, coupled in series with the transistor Q1 (both transistors are in common base configuration), and an extra capacitor, C3. The lower stage based on the Q2 acts as a current source for the upper stage based on the Q1, of both the $\mathrm{DC}$ and the $\mathrm{AC}$ signals. The emitter of the $\mathrm{Q} 1$ is supplied with the feedback signals $V_{C 2}$ and $V_{C 2+C 3}$ from the capacitor $\mathrm{C} 2$ via the Q2 and directly from the capacitor C3.
The fundamental frequency can be estimated as

$$
\omega^{*}=2 \pi f^{*}=\sqrt{\frac{C 1 C 2+C 1 C 3+C 2 C 3}{L C 1 C 2 C 3}}
$$
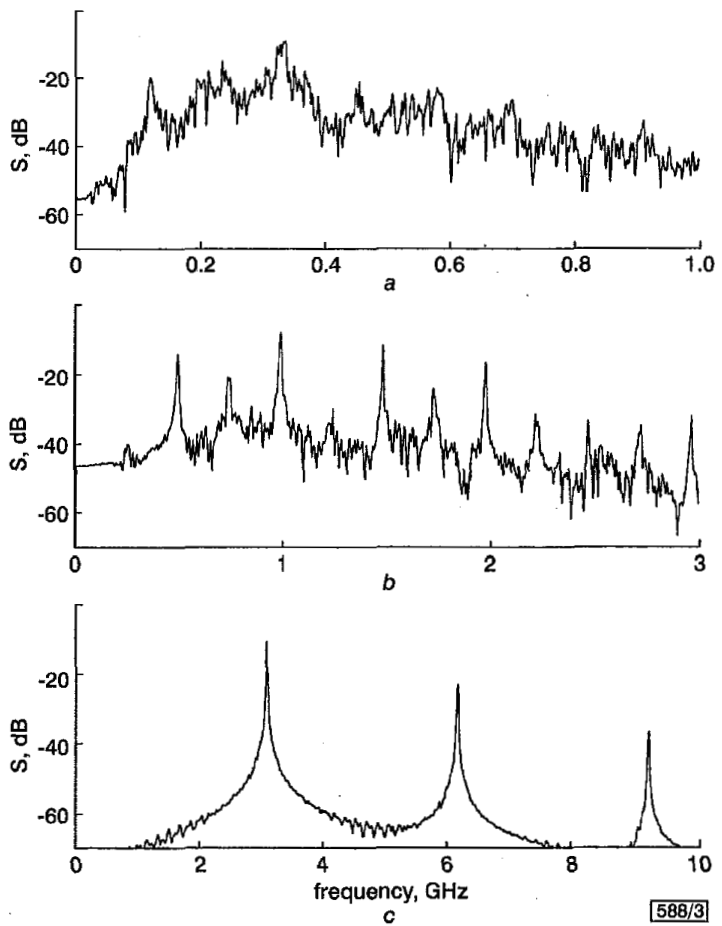

Fig. 3 Power spectra of $V_{K}$ from classical Colpitts oscillator $V 0=15 \mathrm{~V}, 10=20 \mathrm{~mA}$

$a f^{*}=300 \mathrm{MHz}, R=28 \Omega, L=25 \mathrm{nH}, C l=C 2=20 \mathrm{pF}$

$b f^{*}=1 \mathrm{GHz}, R=25 \Omega, L=8 \mathrm{nH}, C 1=C 2=5 \mathrm{pF}$

$c f^{*}=3 \mathrm{GHz}, R=25 \Omega, L=2.5 \mathrm{nH}, C \mathrm{l}=1 \mathrm{pF}, C 2=0.5 \mathrm{pF}$
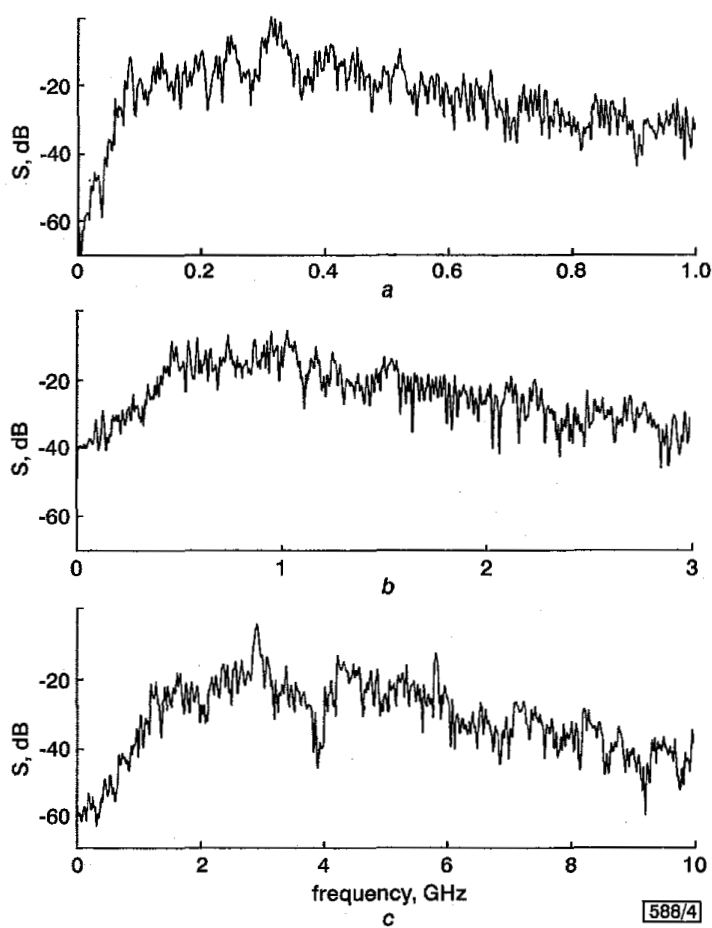

Fig. 4 Power spectra of $V_{K I}$ from two-stage Colpitts oscillator $V 1=30 \mathrm{~V}, V 2=15 \mathrm{~V}, 10=30 \mathrm{~mA}$

$a f^{*}=300 \mathrm{MHz}, R=25 \Omega, L=25 \mathrm{nH}, C 1=15 \mathrm{pF}, C 2=C 3=47 \mathrm{pF}$ $b f^{*}=1 \mathrm{GHz}, R=34 \Omega, L=8 \mathrm{nH}, C 1=4 \mathrm{pF}, C 2=C 3=15 \mathrm{pF}$ $c f^{*}=3 \mathrm{GHz}, R=20 \Omega, L=2 \mathrm{nH}, C l=1 \mathrm{pF}, C 2=C 3=3 \mathrm{pF}$ 
Results: To compare the features of the classical Colpitts oscillator and of the novel chaotic oscillator, simulations of the circuits in Figs. 1 and 2 were performed by means of the Electronics Workbench Professional simulator, based on the PSpice software. The BFG520 type BJTs with $f_{T}=9 \mathrm{GHz}$ were used in both circuits. The simulation results are shown in Figs. 3 and 4 . The fundamental frequency $f^{*}$ of the chaotic oscillations for the classical Colpitts oscillator was not higher than $1 \mathrm{GHz}$. Meanwhile, the $f^{*}$ as high as $3 \mathrm{GHz}$ can be achieved with the same type of BJT in the novel oscillator, i.e. $f^{*}$ could be increased by a factor of three.

This result was confirmed experimentally with the 2N3904 type BJTs having similar characteristics to the above-mentioned $2 \mathrm{~N} 2222 \mathrm{~A}$ transistors. The $f^{*}$ could be shifted from $25 \mathrm{MHz}$ in the classical chaotic Colpitts oscillator to $90 \mathrm{MHz}$ in the novel one.

Conclusion: The suggested modification of the chaotic Colpitts oscillator allows shifting of the fundamental frequencies to higher ones, closer to the threshold frequencies of the transistors.

Acknowledgment: The work of A. Tamaševičius, G. Mykolaitis, A. Cenys and A.N. Anagnostopoulos was supported in part by NATO under Contract No. PST.CLG 977018.

(C) IEE 2001

Electronics Letters Online No: 20010398

29 January 2001

DOI: 10.1049/el:20010398

A. Tamaševičius, G. Mykolaitis, S. Bumelienè and A. Čenys A. Tamasevicius, G. Mykolaitis, S. Bumelienè and A. Cenys
(Semiconductor Physics Institute, A. Goštauto 11, LT-2600 Vilnius, Lithuania)

E-mail: tamasev@uj.pfi.1t

A.N. Anagnostopoulos (Department of Physics, Aristotle University of Thessaloniki, GR-54006 Thessaloniki, Greece)

E. Lindberg (Department of Information Technology, 344, Technical University of Denmark, DK-2800 Lyngby, Denmark)

G. Mykolaitis: Also with the Vilnius Gediminas Technical University, Sauletékio 7, LT-2054 Vilnius, Lithuania

\section{References}

1 KENNEDY, M.P.: 'Chaos in the Colpitts oscillator', IEEE Trans. Circuits Syst. I, 1994, CAD-41, (11), pp. 771-774

2 LINDBERG, E.: 'Colpitts, eigenvalues and chaos'. Proc. 5th Int. Workshop NDES'97, Moscow, Russia, June 1997, pp. 262-267

3 MAGGIO, G.N., FEO, O.DE., and KENNEDY, M.P.: 'Nonlinear analysis of the Colpitts oscillator and applications to design', IEEE Trans. Circuits Syst. I, 1999, CAD-46, (9), pp. 1118-1130

4 WEGENER, C., and KENNEDY, M.P.: 'RF chaotic Colpitts oscillator'. Proc. 3rd Int. Workshop NDES'95, Dublin, Ireland, July 1995, pp. $255 \sim 258$

5 PANAS, A., KYARGINSKY, B., and MAXIMOV, N.: 'Single-transistor microwave chaotic oscillator'. Proc. Int. Symp. NOLTA'2000, Dresden, Germany, Sept. 2000, pp. 445-448

\section{Fault tolerant computation of large inner products}

\section{Imbert and G.A. Jullien}

A new technique for applying fault tolerance to modulus replication RNS computations by adding redundancy to the independent computational channels is introduced. This technique provides a low-overhead solution to fault tolerant large inner product computations.

Introduction: In the MRRNS system, numbers are represented as polynomials of indeterminates which are powers of 2. As an example, if we use the indeterminate $x=8$, we have

$$
79=x^{2}+x+7=x^{2}+2 x-1=2 x^{2}-6 x-1=\cdots
$$

The MRRNS system makes use of the fact that every polynomial of degree $n$ can be uniquely defined by its values at $n+1$ distinct points, and that closed arithmetic operations can be performed over completely independent channels [1]. Another advantage of using the reduced dynamic range channels is the ability to implement the arithmetic over finite fields $G F(p)[2]$. If we choose $p$ as a
Fermat prime, i.e. a number of the form $2^{2^{n}}+1$, modular multiplication can be much simplified [3].

As in [4] we express our algorithm in term of matrix transformations and denote $M_{p}$ and $M_{p}^{-1}$ as the matrices used for the evaluation and the interpolation steps, respectively. In the following, we shall use $m_{k}$ to denote the vector composed of the $k$ th row of $M_{p}^{-1}$, and $S$ for the set of $N$ distinct points. It is important that $N$ must be large enough, not only to represent the input polynomials, but more importantly the result of the computation(s).

The technique is very efficient for computing many additions and multiplications in between the mapping and recovery stages, particularly where we can restrict the number of cascaded multiplications to one in any signal flow path. Fortunately, inner product computations, which are heavily used in DSP algorithms, allow this restriction for an arbitrary inner product length.

The advantages of implementing VLSI include reducing the interconnect span in the computational data path; this leads to easier testing and lower power [3]. Since the computations are independent we can also purposely skew the clocks between the independent data paths, thus reducing the clock current spike.

One of the potential advantages of independent computations is the ability to perform fault detection and correction at a much reduced complexity compared to more classical computational fault tolerant techniques. Although this has been previously explored at the circuit level [5], no one has yet taken advantage of the algebraic structure of the MRRNS. In this Letter we open the exploration of a new technique for fault tolerance using the independent computational structure of the MRRNS system.

Error detection: The detection technique we propose is based on being able to easily compute the constant term of the final polynomial since it depends only on the constant term of each of the initial polynomials involved in the computation.

Definition 1: If $u, v$ are two vectors in $G F(p)^{n}$, we denote $d(u, v)$ the distance between $u$ and $v$ as the number of coordinates in which $u$ and $v$ differ.

Thus, if $u$ is the correct result and $v$ is the vector obtained after the inner product, $d(u, v)$ gives the number of errors. This definition can be seen as an extension of the Hamming distance used in classical coding theory. The following theorem can be used to detect a single error.

Theorem 1: Let $w, z$ be the correct and computed vectors, respectively, and let $Q(x)=q_{0}+q_{1} x+q_{2} x^{2}+\ldots+q_{n} x^{n}$ be the final polynomial. Let us assume $0 \notin m_{1}$ (this is the case if $0 \notin S$ ), and $d(w, z) \leq 1$, i.e. that at most one error occurred. Then $d(w, z)=1$ iff $m_{1} \cdot z \neq q_{0}$

Proof: See [6].

Error correction: Let us consider the following example. We compute the inner product $79 \times 47+121 \times 25=6738$ with correction for one channel in error. We first define four polynomials that correspond to the values $79,47,121,25$; let the indeterminate $x=$ 8 , and assign the following polynomial representations: $79 \rightarrow-1+$ $2 x+x^{2}, 47 \rightarrow-1+6 x, 121 \rightarrow 1+7 x+x^{2}$ and $25 \rightarrow 1+3 x$. Since the final polynomial is third-order, four distinct points are sufficient for its representation, but to correct for one error, we add a redundant channel; i.e. we compute over five points. We will let $S$ $=\{-1,1,-2,2,3\}$. If we express the evaluation step in terms of matrix operations, the vectors composed of the polynomial coefficients now have five co-ordinates, the last one clearly being equal to 0 . We also assume that the coefficients of the final polynomial belong to the set $\{-128, \ldots, 128\}$, which allows us to compute over $G F(257)$. Evaluating the polynomials at these five points gives the vectors $u_{1}=(-2,2,-1,7,14), v_{1}=(-7,5,-13,11,17), u_{2}=(-5,9$, $-9,19,31)$ and $v_{2}=(-2,4,-5,7,10)$. The component-wise operations then give: $w_{1}=u_{1} \otimes v_{1}=(14,10,13,77,-19), w_{2}=u_{2} \otimes v_{2}$ $=(10,36,45,-124,53)$ and $w=w_{1} \oplus w_{2}=(24,46,58,-47,34)$. The final result is recovered by computing

$$
\begin{aligned}
q & =M_{257}^{-1} \times w \\
& =\left[\begin{array}{ccccc}
-128 & 1 & 77 & 128 & -77 \\
85 & -85 & -107 & 107 & 0 \\
75 & -22 & -107 & 22 & 32 \\
43 & -43 & 107 & -107 & 0 \\
-75 & -107 & 30 & 107 & 45
\end{array}\right] \times\left[\begin{array}{c}
24 \\
46 \\
58 \\
-47 \\
34
\end{array}\right] \\
& =(2,2,33,9,0)
\end{aligned}
$$

